\title{
Burden of eye conditions at a specialised eye hospital in KwaZulu-Natal, South Africa
}

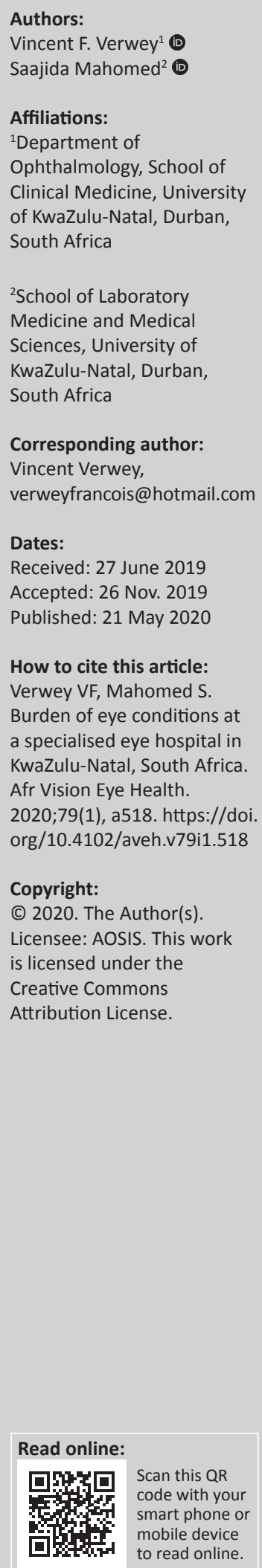

Background: Comprehension of the burden of eye diseases in an area is essential for adequate budgeting and resource allocation.

Aim: The aim of the study was to describe the epidemiology of the presenting eye conditions at a provincial eye hospital.

Setting: A retrospective audit was conducted of first-time presenting patients at the McCord Provincial Eye Hospital over a 6-month period.

Methods: This was an observational, analytic cross-sectional study. Patients' basic demographic and diagnoses were captured. Categorical variables were summarised using frequencies and percentages. Age was summarised using the mean and standard deviation. Differences in the number of patients seen per month, week and day of the week were compared using analysis of variance.

Results: A total of 2250 new patients were seen over the 6-month period. There were more females $(n=1253,55.7 \%)$ than males $(n=997,44.3 \%)$. There were $186(8.3 \%)$ patients $(\leq 14$ years). The three most common presenting conditions among adults were cataract $(n=743,36.0 \%)$, posterior segment disorders $(n=397,19.2 \%)$ and glaucoma $(n=261,12.6 \%)$. Regarding posterior segment disorders, diabetic retinopathy was the commonest condition ( $n=284,71 \%)$, followed by retinal detachment $(n=34,8.5 \%)$. Among the paediatric patients, the most common condition was squint $(n=55,29.6 \%)$, followed by orbital conditions $(n=29$, $15.6 \%)$ and cataract $(n=25,13.4 \%)$.

Conclusion: Audits of the burden of eye conditions provide useful information for human resource management; appropriate allocation, as well as availability of ophthalmologic equipment and staff, can be guided by making use of such information. Further studies and surveillance of eye conditions are needed to plan for better eye health services for patient care.

Keywords: eye conditions; cataract; South Africa.

\section{Introduction}

Eye health is an important aspect of healthcare services. Many eye conditions require access to specialised care, and they can lead to blindness if left untreated. Limited information is available regarding the burden of eye conditions in South Africa, as data on the burden of eye conditions presenting to public or private health facilities are not routinely collected or reported. Data from the 1980s and 1990s show that the major causes of blindness and impaired vision in South Africa were cataract, glaucoma, refractive errors and corneal scarring. ${ }^{1}$ In 2002, a study in Limpopo reported a prevalence of blindness of $0.7 \%$, with the main causes being cataract, glaucoma, corneal opacities and trauma. ${ }^{2}$ Refractive errors remain the leading cause of visual impairment worldwide. ${ }^{3}$

Human resources for eye health need to be planned according to the epidemiology of eye conditions, in terms of the requirements for diagnosis and treatment. ${ }^{4}$ A good understanding of the burden of eye diseases in an area is essential for adequate budgeting and resource allocation. The VISION 2020 Global Initiative by the World Health Organization and the International Agency for the Prevention of Blindness emphasise the importance of human resources, and the implementation and success of this programme to reduce avoidable causes of visual impairment. ${ }^{4}$ In sub-Saharan Africa, poor quality and lack of availability of data regarding human resources in eye health impede planning and implementation of programmes to prevent blindness. In a review of human resources for eye health among 21 countries in 
sub-Saharan Africa in 2014, South Africa was excluded because of insufficient data regarding the number of various practitioners dealing with eye health. ${ }^{5}$

The demographic transition in sub-Saharan Africa and an increase in the number of diabetic patients are important factors while dealing with eye health. An increase in the prevalence of diabetic retinopathy, age-related macular degeneration and glaucoma in a population with increasing life expectancy has implications on the resources required to provide eye health services. ${ }^{6,7}$

Visual impairment has been linked to poverty, decreased autonomy and social interaction, mental health disorders, such as depression, as well as a decline in physical mobility and self-care. ${ }^{8,9,10}$ All of these factors contribute to a decline in the quality of life and socio-economic interaction.

Many cases of blindness can be prevented by providing timely access to appropriate eye healthcare services. Blindness and visual impairment can result in a lack of education, unemployment and can be a burden on families and the healthcare systems. ${ }^{11}$

\section{Objectives}

The aim of this study was to describe the epidemiology of eye conditions at a provincial hospital, and to determine if there are any differences in the number of patients seen per day of the week.

\section{Methods}

The study was conducted at the McCord Provincial Eye Hospital (MPEH), which is the only specialised eye hospital in the province of KwaZulu-Natal (KZN), South Africa. This hospital serves the majority of patients requiring healthcare from eThekwini, iLembe and Ugu health districts. The services offered at MPEH are structured in a systematic manner to help streamline the patient flow. First-time patients without appointments, as well as referred patients, are first triaged at the primary eye care unit (PECU), where they are screened by a trained ophthalmic nurse. Patients assessed as having a primary eye care condition are referred to either a medical officer, an ophthalmic nurse or an optometrist. Patients who are assessed as having signs and symptoms that warrant a second triage are sent to an ophthalmologist for further assessment. During this second triage, patients requiring prompt intervention are referred to the 'intake team' for further management. This includes patients presenting with ophthalmic emergencies, cataracts, vision-threatening conditions and all eye conditions requiring intervention and a higher level of care, as compared to that provided at the PECU. The intake team assesses these patients, and they are either admitted or treated as outpatients. The intake team comprises a consultant, registrar and medical officers.
This was an observational, analytic, cross-sectional study. A retrospective chart review was conducted for patients who presented to the intake team at MPEH over a 6-month period, from May to October 2016, as first presentations requiring treatment. The exclusion criteria were: (1) any follow-up patients seen by the intake team (patients previously known to $\mathrm{MPEH}$ ) or (2) patients presenting to the intake team before 08:00 or after 16:00 on weekdays or patients seen over the weekend.

Patients' basic demographic data (gender and age) and diagnoses were recorded using Microsoft Excel spread sheet. The diagnoses were categorised into 11 broad groups of eye conditions:

- cataract (age-related, congenital or secondary to other conditions)

- vascular disease (vein or artery occlusions)

- conjunctival pathology (pterygiums, conjunctival masses)

- neurologic eye conditions (ptosis, cranial nerve palsies)

- orbit (thyroid eye disease, proptosis, idiopathic orbital inflammatory disease, orbital masses, infections)

- trauma (lid, orbital or globe)

- squints (esotropia or exotropia)

- uveitis (anterior, intermediate, posterior segment or panuveitis)

- glaucoma

- corneal pathology (ulcers, keratoconus or dystrophies)

- posterior segment disorders (retinal detachment, diabetic retinopathy requiring urgent intervention and other retinopathies).

The data were analysed using the Statistical Package for Social Sciences (SPSS) version 25. Categorical variables were summarised using frequencies and percentages. Age was summarised using the mean and standard deviation (SD). Patients were categorised as paediatric patients if they were below the age of 15 years. ${ }^{12,13}$ Differences in the number of patients seen per month, week and day of the week were compared using analysis of variance (ANOVA). Results were considered significant for $p<0.05$.

Gatekeeper permission was obtained from the hospital management and the KwaZulu-Natal Department of Health. Institutional ethics approval was obtained from the Biomedical Research and Ethics Committee at the University of KwaZulu-Natal.

\section{Ethical considerations}

Ethical approval to conduct the study was obtained from the University of KwaZulu-Natal Biomedical Research Ethics Committee (clearance number: BE 453/16).

\section{Results}

A total of 2250 new patients were seen by the intake teams over the 6-month period. There were more females $(n=1253$, $55.7 \%)$ than males $(n=997,44.3 \%)$. The mean age of females (54.2 years, SD 22.3) was significantly higher than the mean 
TABLE 1: Gender of patients presenting to the McCord Provincial Eye Hospital, May-October 2016.

\begin{tabular}{|c|c|c|c|c|c|c|}
\hline \multirow[t]{2}{*}{ Month } & \multicolumn{2}{|c|}{ Male } & \multicolumn{2}{|c|}{ Female } & \multicolumn{2}{|c|}{ Month total } \\
\hline & $n$ & $\%$ & $n$ & $\%$ & $n$ & $\%$ \\
\hline May & 153 & 42 & 212 & 58 & 365 & 100 \\
\hline June & 190 & 42.4 & 258 & 57.6 & 448 & 100 \\
\hline July & 179 & 45.7 & 213 & 54.3 & 392 & 100 \\
\hline August & 199 & 45.1 & 242 & 54.9 & 441 & 100 \\
\hline September & 124 & 47.7 & 136 & 52.3 & 260 & 100 \\
\hline October & 152 & 44.2 & 192 & 55.8 & 344 & 100 \\
\hline Gender total & 997 & - & 1253 & - & 2250 & - \\
\hline
\end{tabular}

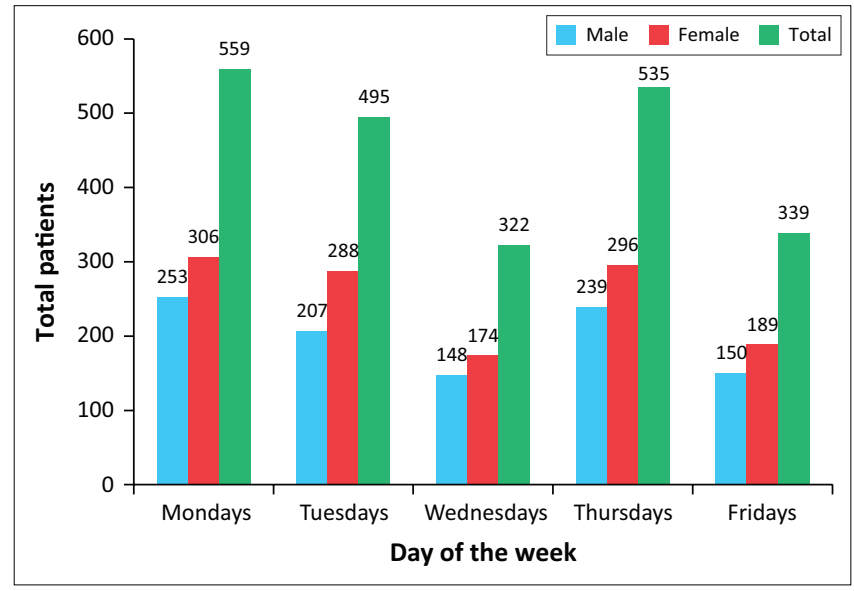

FIGURE 1: The number of patients seen per day of the week at the McCord Provincial Eye Hospital, May 2016 - October 2016.

age of males (46.6 years, SD 21.7) $(p=0.005)$. There were $186(8.3 \%)$ paediatric patients $(\leq 14$ years $)$.

The number of patients seen per month ranged from 260 in September to 485 in June (Table 1). There was no significant difference in the number of patients seen per week of the month. The mean number of patients seen per day of the week differed, with a significantly higher number of patients seen on Mondays, Tuesdays and Thursdays, compared to Wednesdays and Fridays $(p<0.05)$ (Figure 1$)$. The highest number of patients were seen on Tuesdays $(p=0.025)$ and the least number of patients were seen on Fridays $(p=0.046)$.

The three most common presenting conditions for each month during the 6-month period were cataract $(n=768$, $34.1 \%)$, posterior segment disorders $(n=17.8 \%)$ and glaucoma ( $n=268,11.9 \%$ ). The mean number of patients presenting with cataracts was significantly higher than the number of patients presenting with other conditions $(p<0.05)$. The majority of cataract cases were age related $(n=661,86 \%)$. Among the patients with age-related cataracts, two-thirds $(n=437,66.1 \%)$ were females. Twenty-three $(3 \%)$ patients had congenital cataracts, and $84(11 \%)$ patients had cataracts secondary to other causes. In the category of posterior segment disorders, diabetic retinopathy was the commonest condition $(n=284,71 \%)$, followed by retinal detachment $(n=34,8.5 \%)$. A total of $141(6.3 \%)$ patients presented with trauma-related eye conditions, of which the majority were males $(n=108,76.6 \%)$ (Table 2$)$.
TABLE 2: Eye conditions seen at the McCord Provincial Eye Hospital, May-October 2016.

\begin{tabular}{|c|c|c|c|}
\hline Conditions & Male & Female & Total \\
\hline Cataract & - & - & 768 \\
\hline Age-related & 224 & 437 & 661 \\
\hline Congenital & 15 & 8 & 23 \\
\hline Secondary to other conditions & 47 & 37 & 84 \\
\hline Posterior segment disorders & - & - & 400 \\
\hline Diabetic retinopathy & 111 & 173 & 284 \\
\hline Retinal detachment & 12 & 22 & 34 \\
\hline Other & 38 & 44 & 82 \\
\hline Glaucoma & 123 & 145 & 268 \\
\hline Orbit & - & - & 146 \\
\hline Thyroid eye disease & 2 & 3 & 5 \\
\hline Proptosis & 10 & 3 & 13 \\
\hline Idiopathic orbital inflammatory disease & 1 & 0 & 1 \\
\hline Orbital masses & 9 & 19 & 28 \\
\hline Orbital inflections & 16 & 13 & 29 \\
\hline Other & 32 & 38 & 70 \\
\hline Trauma & - & - & 141 \\
\hline Lid & 33 & 8 & 41 \\
\hline Orbital & 15 & 7 & 22 \\
\hline Globe & 46 & 13 & 59 \\
\hline Other & 14 & 5 & 19 \\
\hline Conjunctival pathology & - & - & 127 \\
\hline Pterygiums & 7 & 21 & 28 \\
\hline Conjunctival masses & 25 & 19 & 44 \\
\hline Other & 19 & 36 & 55 \\
\hline Corneal pathology & - & - & 126 \\
\hline Ulcers & 45 & 30 & 75 \\
\hline Keratoconus & 12 & 12 & 24 \\
\hline Dystrophies & 1 & 1 & 2 \\
\hline Other & 13 & 12 & 25 \\
\hline Uveitis & - & - & 87 \\
\hline Anterior & 26 & 20 & 46 \\
\hline Intermediate & 1 & 1 & 2 \\
\hline Posterior & 8 & 18 & 26 \\
\hline Panuveitis & 10 & 3 & 13 \\
\hline Neurologic presentation & - & - & 82 \\
\hline Ptosis & 6 & 6 & 12 \\
\hline Cranial nerve palsies & 5 & 8 & 13 \\
\hline Other & 20 & 37 & 57 \\
\hline Squints & - & - & 67 \\
\hline Esotropia & 31 & 31 & 62 \\
\hline Exotropia & 2 & 2 & 4 \\
\hline Other & 0 & 1 & 1 \\
\hline Vascular disease & - & - & 38 \\
\hline Vein occlusions & 14 & 16 & 30 \\
\hline Artery occlusions & 2 & 2 & 4 \\
\hline Other & 1 & 3 & 4 \\
\hline
\end{tabular}

Note: Bold data gives the total of the specific category of eye conditions that is listed.

Among the paediatric patients, the most common condition was squint ( $n=55,29.6 \%$ ), followed by orbital conditions $(n=29,15.6 \%)$ and cataract $(n=25,13.4 \%)$.

\section{Discussion}

This is the first report of the epidemiology of eye conditions of patients presenting at a specialised eye hospital in South Africa. Females presented more commonly than males, in keeping with the general demography of users of healthcare services. ${ }^{14,15}$ However, studies from high- and low-income countries, such as the United States, Kenya and 
Bangladesh, reported a higher proportion of males utilising eye care services. ${ }^{16,17,18,19,20,21}$

We found that the females in this study were significantly older than the males. Worldwide, there is an increase in life expectancy, with women living longer. ${ }^{22}$ This is also reflected in South Africa where the life expectancy in females is 67.3 years, compared to 61.1 years in males. ${ }^{13}$ In our study, more females presented with age-related cataracts, and more males presented with trauma-related conditions. This can also explain the age difference between the presenting males and females.

The number of patients was significantly less on Wednesdays and Fridays, compared to the other weekdays. The Department of Ophthalmology has an academic meeting on Wednesday afternoons during which there are registrar teaching sessions, including tutorials, journal club, subspecialist clinics and academic case discussions. Academic ward rounds are held on Friday afternoons. Some referring hospitals are aware of the academic activities and therefore limit patient referrals on these days. Patients choosing not to present to hospitals prior to a weekend could possibly be an explanation for the lower number of patients seen on Fridays. Studies conducted in Portugal and Australia also reported higher non-attendance of patients for general outpatient and gynaecology appointments on Fridays. ${ }^{23,24}$

However, a review of the patterns and influences of healthcare attendance behaviour found no clear and consistent association between day of the week and patient attendance at clinics. ${ }^{25}$

Our finding of cataract being the commonest presenting condition is in keeping with this condition being the leading cause of blindness globally and in South Africa. Uncorrected refractive errors and cataracts remain the leading cause of preventable blindness in lower-middle-income countries. ${ }^{26}$ This is also in keeping with previous studies conducted in South Africa. ${ }^{1,2,11,27}$ Patients in the eThekwini health district are largely dependent on cataract services provided at $\mathrm{MPEH}$, with only Prince Mshiyeni Memorial Hospital (another public hospital) providing the facility of routine cataract surgery in this district. In addition, complicated cataract cases from other districts are referred to MPEH. The low proportion (3\%) of congenital cataracts in this study is because the majority of patients with this condition are being referred directly to a specialised paediatric clinic at Inkosi Albert Luthuli Central Hospital.

Strikingly, a large proportion of patients who presented with posterior segment pathology had diabetic retinopathy. This finding is in contrast to global trends of blindness and visual impairment, for which cataract, age-related macular degeneration and glaucoma are the leading causes. ${ }^{28,29}$ Our finding of a high prevalence (12.6\%) of patients with diabetic retinopathy that required specialised eye care services is almost 1.5 times that of the global estimate (8.5\%). ${ }^{30}$ While there are no national figures available for the prevalence of diabetic retinopathy in South Africa, a study in Cape Town reported that diabetic retinopathy contributed to $8 \%$ of blindness and $11 \%$ of visual impairment, respectively. ${ }^{31}$ The prevalence of diabetes in KZN (12.5\%) is higher than the national prevalence $(9.2 \%),{ }^{32}$ which may explain this high prevalence. Increased predisposition to diabetes in the Indian population (which accounts for $7.4 \%$ of the KZN population $)^{13,20}$ has been described, along with an increase in the prevalence of diabetes among the black South African population. ${ }^{13,33}$ This study included only patients with diabetic retinopathy requiring an intervention (laser, intravitreal injection or surgery); therefore, the prevalence of diabetic retinopathy among this population may be overestimated.

The patients with diabetic retinopathy in our study presented to MPEH for the first time. This suggests that patients with diabetes mellitus are not being routinely screened for diabetic retinopathy. Screening for diabetic retinopathy should be conducted annually at primary healthcare clinics, and patients should be referred to specialist ophthalmic services timely. The importance of primary medical checkup, as well as eye care, for patients with diabetes mellitus must be stressed. Improvements in primary healthcare and screening for target organ damage will result in better prognosis for these patients. In the long-term, better glucose control and risk factor management will result in improved quality of life and preservation of vision.

The effect of glaucoma on vision, peripheral vision in particular, is profound in advanced glaucoma. More than $10 \%$ of the patients in this study had glaucoma that required intervention. South Africa does not have a formal screening protocol for glaucoma, which may result in many patients being diagnosed with the advanced disease.

\section{Limitations}

This study included only those patients seen on weekdays and during normal working hours. The exclusion of patients seen after-hours and on weekends can skew the epidemiology of the conditions seen. This is particularly true for traumarelated cases, which explains the low proportion of patients with ocular trauma. As this was a descriptive study, we were unable to assess risk factors for any of the conditions. We were also unable to categorise the severity of diagnosed diseases, such as diabetic retinopathy and glaucoma, based on the data used for this study. During the 6-month study period, there were strict screening criteria in place for the referral of patients to the intake team because of doctor shortages, which would have resulted in an underestimation of the prevalence of the various conditions requiring interventions.

\section{Conclusion}

Audits of the burden of eye conditions provide useful information for human resource management. Appropriate allocation, as well as availability of ophthalmologic equipment and staff, can be guided by using such information. 
The high number of patients with cataracts highlights the need to increase resource allocation in order to meet the needs of this population. Screening for glaucoma and diabetic retinopathy should be implemented and improved at a primary healthcare level. The importance of screening cannot be underestimated, as timeous management and prevention of complications of these conditions can prevent blindness and decrease the long-term financial burden on the health system. Patients need to be educated on these conditions, so that they can present themselves for screening. Further studies and surveillance of eye conditions are needed at the district and provincial levels to plan for better eye health services for patient care.

\section{Acknowledgements \\ Competing interests}

The authors have declared that no competing interest exist.

\section{Authors' contributions}

V.F.V. prepared the protocol, collected the data and wrote the manuscript. S.M. supervised the writing of the protocol, analysed the data and assisted with the preparation of the final manuscript.

\section{Funding information}

This research received no specific grant from any funding agency in the public, commercial or not-for-profit sectors.

\section{Data availability statement}

Data sharing is not applicable to this article.

\section{Disclaimer}

The views and opinions expressed in this article are those of the authors and do not necessarily reflect the official policy or position of any affiliated agency of the authors.

\section{References}

1. Bucher PJ, ljsselmuiden CB. Prevalence and causes of blindness in the northern Transvaal. Br J Ophthalmol. 1988;72(10):721-726. https://doi.org/10.1136/bjo. 72.10.721

2. Cook CD, Knight SE, Crofton-Briggs I. Prevalence and causes of low vision and blindness in northern KwaZulu. S Afr Med J. 1993;83(8):590-593.

3. Courtright $P$, Mathenge $W$, Kello $A B$, Cook C, Kalua K, Lewallen $S$. Setting targets for human resources for eye health in sub-Saharan Africa: What evidence should be used? Hum Resour Health. 2016;14, Article ID 11. https://doi.org/10.1186/s12960016-0107-x

4. VISION2020. Global Initiative for the elimination of avoidable blindness: Action plan 2006 to 2011 [homepage on the Internet]. Geneva: WHO; 2007 [cited 2019

5. Palmer JJ, Chinanayi F, Gilbert A, et al. Mapping human resources for eye health in 21 countries of sub-Saharan Africa: Current progress towards VISION 2020. Hum Resour Health. 2014;12, Article ID 44. https://doi.org/10.1186/1478-4491-12-44

6. Bourne RRA, Stevens GA, White RA, et al. Causes of vision loss worldwide, 1990-2010: A systematic analysis. Lancet Glob Health. 2013;1(6):e339-e349. https://doi.org/10.1016/S2214-109X(13)70113-X

7. Mash B, Powell D, Du Plessis F, Van Vuuren U, Michalowska M, Levitt N. Screening for diabetic retinopathy in primary care with a mobile fundal camera: Evaluation of a South African pilot project. S Afr Med J. 2007;97(12):1284-1288.

8. Lamoureux EL, Fenwick E, Moore K, et al. Impact of the severity of distance and near vision impairment on depression and vision specific quality of life in olde people living in residential care. Invest Ophthalmol Vis Sci. 2009;50(9):4103-4109. https://doi.org/10.1167/iovs.08-3294
9. Bekibele $\mathrm{CO}$, Gureje O. Impact of self-reported visual impairment on quality of life in the Ibadan study of aging. Br J Ophthalmol. 2008;92(5):612-615. https://doi. org/10.1136/bjo.2007.124859

10. Payot A, Barrington KJ. The quality of life of young children and infants with chronic medical problems: Review of the literature. Curr Probl Pediatr Adolesc Health Care. 2011;41(4):91-101. https://doi.org/10.1016/j.cppeds.2010.10.008

11. Oduntan AO, Nthangeni ME, Ramudzuli R, Madu SN. Causes and prevalence of low vision and blindness in black South African adults in the Limpopo province. S Afr Optom. 2002;62(2):8-15.

12. National Department of Health, South Africa. Age definitions document [homepage on the Internet]. Pretoria: NDoH; c2012 [cited 2019 Nov 05]. Available from: https://www.health.gov.za/index.php/shortcodes/2015-03-29-10-42-47/2015-0610-09-23-36/2015-06-10-09-26-11?download=844:icd-10-definitions.

13. Statistics South Africa. Statistical release P0302 Midy-year population estimates 2018 [homepage on the Internet]. Pretoria: Stats SA; c2018 [cited 2019 Nov 05]. Available from: https://www.statssa.gov.za/publications/P0302/ Nov 05]. Avail
P03022018.pdf.

14. Khan T, Bertram MY, Jina R, Mash B, Levitt N, Hofman K. Preventing diabetes blindness: Cost effectiveness of a screening programme using digital nonmydriatic fundus photography for diabetic retinopathy in a primary health care setting in South Africa. Diabetes Res Clin Pract. 2013;101(2):170-176. https://doi. org/10.1016/j.diabres.2013.05.006

15. Thompson AE. Ansisimowicz Y, Miedema B, Hogg W, Wodchis WP, Aubrey-Bassler $K$. The influence of gender and other patient characteristics in healthcare-seeking behavior: A QUALICOPC study. BMC Health Serv Res 2017;17(1):222. https://doi. org/10/10.1186/s12913-017-2189-0

16. Wang Y, Hunt K, Nazareth I, Freemantle N, Petersen I. Do men consult less than women? An analysis of routinely collected UK general practice data. BMJ Open. 2013;3(8):e003320. https://doi.org/10.1136/bmjopen-2013-003320

17. Channa R, Zafar SN, Canner JK, Haring RS, Schneider EB, Friedman DS. Epidemiology of eye-related emergency department visits. JAMA Ophthalmol. 2016;134(3):312-319. https://doi.org/10.1001/jamaophthalmol.2015.5778

18. Mau M, Alam MS, Akma M, Akter MS, Kabir MH. Pattern of eye diseases in a tertiary hospital in a suburban area: A retrospective study. ORION Med J. 2007;28(1):492-494.

19. House DR, Nyabera SL, Yusi K, Rusyniak DE. Descriptive study of an emergency centre in Western Kenya: Challenges and opportunities. AfJEM. 2014;4(1):19-24. https://doi.org/10/1016/j.afjem.2013.08.069

20. Mohamed AA, Lakho K. Pattern of eye diseases at tertiary eye hospital in Sudan (Makah Eye Hospital, Khartoum) Albasar. Int J Ophthalmol. 2015;3(1):15-18. https://doi.org/10.4103/1858-6538.169304

21. Fenton $\mathrm{S}$, Jackson $\mathrm{E}$, Fenton $\mathrm{M}$. An audit of the ophthalmic division of the accident and emergency department of the Royal Victoria Eye and Ear Hospital Dublin. Ir Med J. 2001;94(9):265-266.

22. Fasih U, Rahman A, Shaik A, Fahmi M, Rais M. Pattern of common paediatric diseases at Spencer Eye Hospital. Pak J Ophthalmol. 2014;30(1):10-14.

23. Costa J, Lima M, Sousa D, et al. Impact of appointment reminders via short message service in a District Hospital. Porto: Universidade do Porto; 2010.

24. Pillai R, Bhangu N, Narayanan M, Yoong W. A demographic study to profile nonattenders at a gynaecology outpatient clinic. J Obstet Gynaecol. 2012;32(2): 156-158. https://doi.org/10.3109/01443615.2011.635228

25. McLean S, Gee M, Booth A, et al. Targeting the use of reminders and notifications for uptake by populations (TURNUP): A systematic review and evidence synthesis. Health Serv Deliv Res. 2014;2(34):1-184. https://doi.org/10.3310/ synthesis.

26. World Health Organization. Global Health Observatory data: Life expectancy. [homepage on the Internet]. 2018 [cited 2019 Apr 16]. Available from: https:// www.who.int/gho/mortality_burden_disease/life_tables/situation_trends/en/.

27. Maake Modjadji M, Oduntan Olalekan A. Prevalence and causes of visual impairment in patients seen at Nkhensani Hospital Eye Clinic, South Africa. Afr J Prim Health Care Fam Med. 2015;7(1):1-6. https://doi.org/10.4102/phcfm. v7i1.728

28. World Health Organization. Visual impairment and blindness 11 October 2018 [homepage on the Internet]. c [cited 2019 Apr 16]. Available from: https://www. who/news-room/fact-sheets/detail/blindness-and-visual-impairment.

29. Flaxman SR, Bourne RRA, Resnikoff $S$ et al. Global causes of blindness and distance vision impairment 1990-2020: A systematic review and meta-analysis. Lancet Glob Health. 2017;5(12):e1221-e1234. https://doi.org/10/1016/S2214-109X(17) 30393-5

30. World Health Organization. Global report on diabetes 2016 [homepage on the Internet]. c [cited 2019 Apr 16]. Available from: https://apps.who.int/iris/bitsream/ handle/10665/204871/978924156527_eng.pdf:jsessionid=A8200775A83F878B6 86319512A1D4425? sequence $=1$.

31. Hofman KJ, Cook C, Levitt N. Preventing diabetic blindness: A priority for South Africa. SAMJ 104(10):661-662. https://doi.org/10.7196/SAMJ.8580

32. Sahadew N, Singaram VS, Brown S. Distribution, incidence, prevalence and default of patients with diabetes mellitus accessing public healthcare in the 11 districts of KwaZulu-Natal, South Africa. SAMJ 106(4):389-393. https://doi.org/10.7196/ SAMJ.2016.v106i4.10143

33. Peer N, Steyn K, Lombard C, et al. Rising diabetes prevalence among urban dwelling black South Africans. PLoS One 2012;7(9):e43336. https://doi/10.1371/ journal.pone.0043336 